\title{
Diagnóstico y percepciones sobre el aprendizaje del idioma inglés
}

Diana Paola López Lozano * Angélica María Sánchez Páez

Artículo de investigación

Fecha de Recepción: 1 junio 2018.

Fecha de Aprobación: 30 septiembre 2018.

\section{Resumen}

Esta ponencia tiene como objetivo mostrar resultados de la fase diagnóstica aplicada a estudiantes de la Institución Educativa San Antonio de Ráquira, sede Mirque, sobre el nivel de inglés que ellos tienen y los gustos e intereses relacionados con el aprendizaje de este idioma. Para el desarrollo de esta investigación se trabaja con un tipo de investigación acción participación donde se intenta cambiar el proceso de enseñanza-aprendizaje del idioma inglés con el uso de las Tic en los estudiantes de esta sede. Se desarrolló la fase diagnóstica que proviene de los resultados de las pruebas Instruimos del primer semestre del año 2017 y de una encuesta diseñada por las autoras.
Los resultados muestran que más de la mitad de los estudiantes se encuentran por debajo del nivel mínimo de inglés(A-) y en cuanto a la encuesta arroja como resultado el gusto por el uso de computadores, la internet y otras herramientas tecnológicas que son de agrado de los estudiantes. Por tanto se puede concluir que se hace necesaria una propuesta didáctica encaminada al mejoramiento del nivel de inglés y que tomando los gustos e intereses de los estudiantes, la incorporación de las Tic ayudaría al desarrollo de este proceso.
Palabras
claves:
aprendizaje, enseñanza, lengua extranjera, tecnologías de la información.

*Dipalolo82@hotmail.com

**Angiemar_9@hotmail. com

Institución Educativa San Antonio de Ráquira

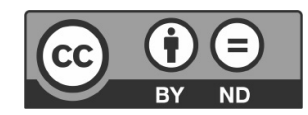




\section{Introducción}

El aprendizaje del idioma inglés resulta en oportunidades superfluo para los estudiantes de la Institución Educativa San Antonio de Ráquira, pues a pesar de ser una necesidad para su entorno turístico, a algunos les parece un proceso complejo; por tal razón es pertinente una propuesta que atienda los intereses, gustos y habilidades de los estudiantes, a partir de una educación flexible, donde cada estudiante sea capaz de construir conocimiento de forma colaborativa.

Con base en lo anterior el aprendizaje de una segunda lengua da al estudiante Raquireño la oportunidad de conocer otras culturas, de aumentar sus posibilidades en el campo laboral, de ampliar sus conocimientos para una vida académica y/o universitaria con muchas más posibilidades de utilización y en general forma estudiantes más ilustrados, participes de las generaciones y de las culturas del nuevo mundo. De Juan González (2012), en su estudio de análisis de artículos académicos muestra resultados positivos acerca del uso de las tecnologías por medio de blog y wikis en la enseñanza del idioma inglés; en su escaneo de artículos se identifican las grandes ventajas y algunas desventajas del uso de las Tic para el aprendizaje de la segunda lengua.

Por otro lado y teniendo en cuenta que hoy día el ser humano está influenciado por un constante cambio respecto al uso de las tecnologías, desarrollar procesos de aprendizaje del idioma inglés a través de los recursos $\mathrm{y}$ herramientas que proporcionan las Tecnologías de la Información y la Comunicación es oportuno.

Diferentes autores como: Jaimes \& Jaimes, Hernández, Parra \& Galván, Rojas, López \& Londoño, Cassany et al., Morchio, Matute, Severín y el ministerio de Educación; resaltan la importancia del aprendizaje del idioma Inglés a través de las Tic. Al respecto Jaimes \& Jaimes (2014) afirman que los docentes colombianos que estén involucrados con las Tic y con el aprendizaje de una segunda lengua como opción laboral, están a la vanguardia del mundo moderno y a la vez establecen las necesidades que el magisterio tiene para poder cumplir las metas que el gobierno propone como mayor capacitación, buenos aulas de bilingüismo actualizadas y docentes con un alto nivel de inglés, capaces de orientar un conocimiento íntegro e innovador a sus estudiantes.

La aplicación de un software específico hace que la clase tenga una secuencia metodológica y un seguimiento del aprendizaje del alumnado coherente y secuencial siguiendo temáticas que correspondan a los diferentes niveles en los Estándares Europeos:

Hernández (2014), realizó una tesis doctoral con el programa B-learning aplicada a más de 400 estudiantes universitarios y logró demostrar que las Tic a través de esa herramienta tecnológica suben el nivel de inglés de los mismos, motivados por el uso de algo novedoso y actualizado. 
Parra \& Galván (2014) y Rojas (2013) implementaron el uso de los recursos de la Web 2.0 (página web, software educativo, videos, audios y blog) y lograron demostrar que el uso de este tipo de herramientas genera un aprendizaje más significativo y motivador sobre todo para estudiantes de grado sexto que están iniciando sus bases en el idioma inglés. Méndez et al. (2015), llevaron a la práctica del aula el software Gran Caco con seis temáticas: colores, números, prendas de vestir, comidas, partes del cuerpo humano, animales y cada una de ellas ofrece diversas actividades que motivaron a los estudiantes a mostrar mejor desempeño en la asignatura y se manifiesta que el proceso enseñanzaaprendizaje mejoró.

"La importancia que tienen las lenguas está determinada por la difusión que éstas tienen, tanto en los medios de comunicación como en la tecnología y la sociedad. El desarrollo que logró EE.UU. de América durante la segunda mitad de este siglo, adicionalmente a la popularización de la televisión, el cine y los medios tecnológicos, impuso el idioma inglés hasta tal punto que se acepta como lengua universal” (López \& Londoño 2012. p 31).

Por otro lado la cartilla Colombia la más educada 2025 en su capítulo Colombia Bilingüe, enfoca el aprendizaje del idioma extranjero en la búsqueda de un avance para aumentar la competitividad y atraer el turismo extranjero. Además adicionan que: "La meta para el año 2018 es subir el nivel
Pre Intermedio B1 del 2\% al 8\% y del 7\% al 35\% de los estudiantes de grado $11^{\circ}$ en nivel Básico A2...El objetivo fundamental es lograr que los estudiantes se comuniquen cada vez mejor en inglés y puedan acceder a mejores oportunidades laborales y profesionales”. (p 67)

El MEN en su preocupación de mejorar el nivel de inglés de los estudiantes de Básica Primaria de las instituciones educativas rurales más lejanas del país, en el año 2011 lanzó su cartilla English

For Colombia "ECO" buscando desarrollar las cuatro habilidades del inglés para ubicar a estos estudiantes en un nivel A1, lo que evidencia que para el gobierno lograr que los estudiantes adquieran un buen nivel de inglés en sus instituciones educativas es aún una prioridad.

El estudiante que comprende otro idioma es capaz de interpretar y hacer lectura crítica en ese mismo idioma, Cassany et al., (2015) asegura que los programas de enseñanza de Lengua Extranjera deben incorporar textos tomados de internet para poder comprender otras culturas y tener una visión más crítica del mundo.

Por su parte, Morchio (2014), en su estudio hace referencia a la apatía que algunos docentes de idiomas sienten al uso de las nuevas tecnologías y más si son dadas por los estudiantes en el aula de clase, manifiesta que es importante darle cabida a las Tic con el propósito de que el estudiante se sienta identificado con la clase de los tiempos actuales.

\footnotetext{
"La importancia que tienen las lenguas está determinada por la difusión que éstas tienen, tanto en los medios de comunicación como en la tecnología y la sociedad. El desarrollo que logró EE.UU. de América durante la segunda mitad de este siglo, adicionalmente a la popularización de la televisión, el cine y los medios tecnológicos, impuso el idioma inglés hasta tal punto que se acepta como lengua universal",
} 
Ahora bien, Matute (2013) en su investigación cuantitativa concluye que algunas instituciones no cuentan con los recursos tecnológicos mínimos que posibilitan la motivación del aprendizaje de otro idioma, aspecto que se toma como desventaja con respecto a otras instituciones, ya que, según su estudio las Tic aumentan el nivel de participación de los estudiantes en esta clase.

Por tanto el impacto de las TIC durante los últimos 30 años ha estado relacionado con la inclusión de las tecnologías, ya que aportan a mejorar la calidad educativa; definiendo la calidad educativa desde el enfoque conductistainstrumental como un conjunto de indicadores mensurables (pruebas) y desde el punto de vista humanista- constructivista focalizado en los procesos de aprendizaje (Severin, 2016).

\section{Método}

Se busca como primera medida acercarse a los estudiantes para conocer y describir sus intereses y necesidades del contexto, para así poder identificar las estrategias que puedan mejorar el proceso de Enseñanza- aprendizaje del idioma Inglés en los estudiantes de la Institución Educativa San Antonio del municipio de Ráquira, Sede Mirque.

Por las características de los medios utilizados para obtener los datos, esta investigación es considerada de campo, pues se concentra en satisfacer las necesidades educativas de los 15 estudiantes de la Sede Mirque, que se describen en la siguiente tabla:

Tabla 1. Caracterización de los participantes

\begin{tabular}{|c|c|c|c|c|c|c|}
\hline Grado & $\begin{array}{c}\text { Total de } \\
\text { estudiantes }\end{array}$ & $\begin{array}{c}\text { Total } \\
\text { hombres }\end{array}$ & Total mujeres & $\begin{array}{c}\text { Edad } \\
\text { promedio }\end{array}$ & $\begin{array}{c}\text { Estrato } \\
\text { promedio }\end{array}$ & $\begin{array}{c}\text { Lugar de } \\
\text { residencia }\end{array}$ \\
\hline Transición & 4 & 3 & 1 & 5 & 1 & Rural \\
\hline Primero & 3 & 1 & 1 & 7 & 2 & Rural \\
\hline Segundo & 3 & 3 & 0 & 7 & 2 & Rural \\
\hline Tercero & 3 & 2 & 1 & 9 & 2 & Rural \\
\hline Cuarto & 2 & 1 & 1 & 9 & 2 & Rural \\
\hline
\end{tabular}

Fuente.-Estudiantes participantes

Análisis. En la anterior tabla se evidencia que hay más hombres que mujeres en esta sede, sus edades oscilan entre 5 y 9 años, el estrato económico predominante es el 2 y todos los estudiantes viven en el área rural.

Acorde con el método utilizado, este estudio es cualitativo, ya que los diferentes datos obtenidos son analizados e interpretados. Para el desarrollo de esta investigación se trabaja con un tipo de investigación acción participación donde se intenta cambiar un fenómeno de interés para un grupo social.

Conforme a los objetivos planteados, esta investigación se desarrolla en cuatro fases, para el caso nos 
centraremos en sólo la primera fase que es la prueba diagnóstica en la que se tomaron los resultados de las pruebas Instruimos año 2017 en su primera etapa, en donde se encuentra que los estudiantes tienen un nivel bajo de Inglés, conocimiento de muy poco vocabulario y en un porcentaje bajo, habilidad para interpretar figuras con algunas palabras que las relacionan.

\section{Prueba diagnóstica}

La prueba aplicada en la primera fase del desarrollo de la propuesta, permite conocer el nivel del idioma inglés de los estudiantes de la Institución Educativa San Antonio, sede Mirque.; la información es analizada e interpretada.

\section{Resultados}

A continuación se presentan los resultados de la fase diagnóstica desarrollada con los estudiantes de la sede Mirque, de la Institución Educativa San Antonio de Ráquira, en donde se analizan cada uno de los datos que arrojan los dos instrumentos tenidos en cuenta: Encuesta y resultados pruebas Instruimos.

Fase Diagnóstica: Esta fase está enmarcada en dos instrumentos, el primero es la aplicación de las pruebas Saber Instruimos diseñadas para los grados de primero a once y son realizadas en dos períodos diferentes en el municipio; el segundo instrumento es una encuesta a todos los estudiantes de la Sede Mirque con el fin de conocer la opinión y los intereses con respecto al aprendizaje del idioma inglés.

\section{Instrumento número uno: Pruebas Saber Instruimos 2017}

En este primer instrumento se tomó como base los resultados de dichas pruebas aplicadas a 11 de los 15 estudiantes matriculados en la sede, de los cuales cuatro se encuentran en grado transición, y las pruebas están diseñadas para los grados de primero a once.

Tabla 2. Puntajes

\begin{tabular}{|c|c|c|c|c|c||}
\hline Estudiante & $\begin{array}{c}\text { Número de } \\
\text { preguntas }\end{array}$ & $\begin{array}{c}\text { Respuestas } \\
\text { correctas }\end{array}$ & $\begin{array}{c}\text { Respuestas } \\
\text { incorrectas }\end{array}$ & Puntaje & Categoría \\
\hline E1 & 8 & 4 & 4 & 50,0 & Medio \\
\hline E2 & 8 & 5 & 3 & 62,50 & Alto \\
\hline E3 & 8 & 2 & 6 & 25,0 & Bajo \\
\hline E4 & 8 & 3 & 5 & 37,50 & Medio \\
\hline E5 & 8 & 5 & 3 & 62,50 & Alto \\
\hline E6 & 8 & 5 & 3 & 62,50 & Alto \\
\hline E7 & 16 & 9 & 7 & 56,25 & Alto \\
\hline E8 & 16 & 5 & 11 & 31,25 & Medio \\
\hline E9 & 16 & 8 & 8 & 50,00 & Medio \\
\hline E10 & 16 & 6 & 10 & 37,50 & Medio \\
\hline E11 & 16 & 6 & 10 & 37,50 & Medio \\
\hline
\end{tabular}

Fuente: Resultados Pruebas Saber Instruimos 
Análisis. De seis estudiantes que se les aplicó la prueba con ocho preguntas, solo tres alcanzaron a contestar correctamente más de la mitad del total de preguntas, los tres estudiantes restantes más de la mitad de respuestas fueron incorrectas. Por otro lado, de cinco estudiantes que se les aplicó la prueba con 16 preguntas, solo uno de ellos tuvo mayor número de respuestas correctas que incorrectas, los demás fueron más las respuestas incorrectas que las correctas y en un sólo caso fue igual en aciertos y desaciertos. Esto indica que la mitad de los estudiantes se encuentran en un desempeño Medio, menos de la mitad en un desempeño Alto y en desempeño Bajo un estudiante.

\section{Tabla 3. Nivel de inglés}

Los resultados de las pruebas Saber Instruimos muestran las competencias y los desempeños de cada uno de los estudiantes los cuales van directamente relacionados con el nivel de inglés que alcanzo cada uno, a continuación se específica dicho resultado.

\begin{tabular}{|c|c|c|}
\hline Estudiante & Puntaje & Nivel \\
\hline E1 & 5,00 & A- \\
\hline E2 & 6,25 & A1 \\
\hline E3 & 2,50 & A- \\
\hline E4 & 3,75 & A- \\
\hline E5 & 6,25 & A1 \\
\hline E6 & 6,25 & A1 \\
\hline E7 & 5,63 & A- \\
\hline E8 & 3,13 & A- \\
\hline E9 & 5,00 & A- \\
\hline E10 & 3,75 & A- \\
\hline E11 & 3,75 & A- \\
\hline
\end{tabular}

Fuente: Resultados Pruebas Saber Instruimos

Análisis. La mayoría de los estudiantes se encuentran en un nivel A-, en donde se interpreta que no alcanzan los resultados esperados y muy pocos estudiantes están en un nivel A1 principiante, donde se infiere que la gran mayoría de los estudiantes no reconocen vocabulario básico y tienen dificultades para formar oraciones sencillas, con estructuras básicas según los parámetros dados por los
Estándares Europeos analizados desde el ministerio de educación (MEN 2006).

\section{Instrumento número dos: aplicación de encuestas}

Este instrumento consta de cinco preguntas cerradas y una abierta en donde los estudiantes pueden contestar de forma libre según su preferencia o interés. 


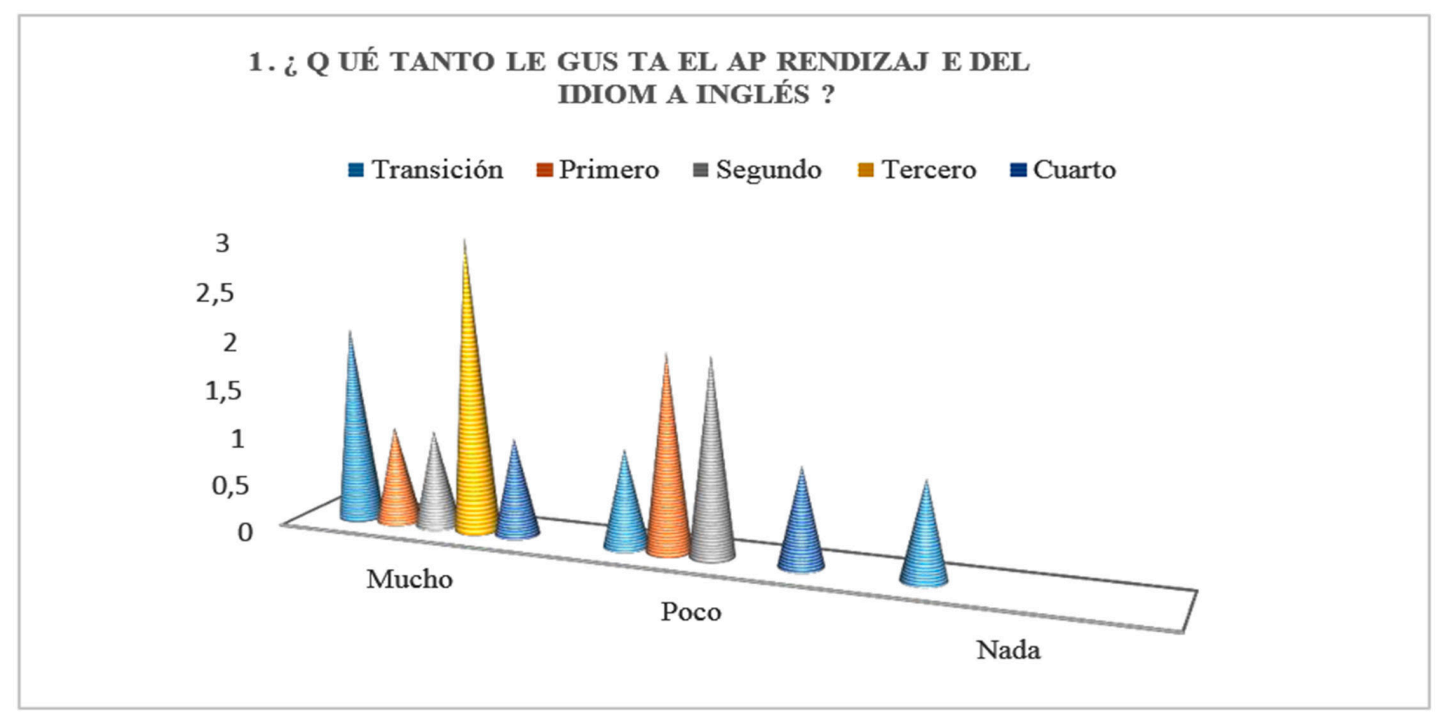

Gráfico 1. Gusto por el idioma. Fuente. Elaboración propia

Análisis. En la pregunta más de la mitad respondieron que el aprendizaje del idioma inglés les gusta mucho, menos de la mitad indicaron que poco les gusta y en una cantidad mínima respondió que nada le gusta.

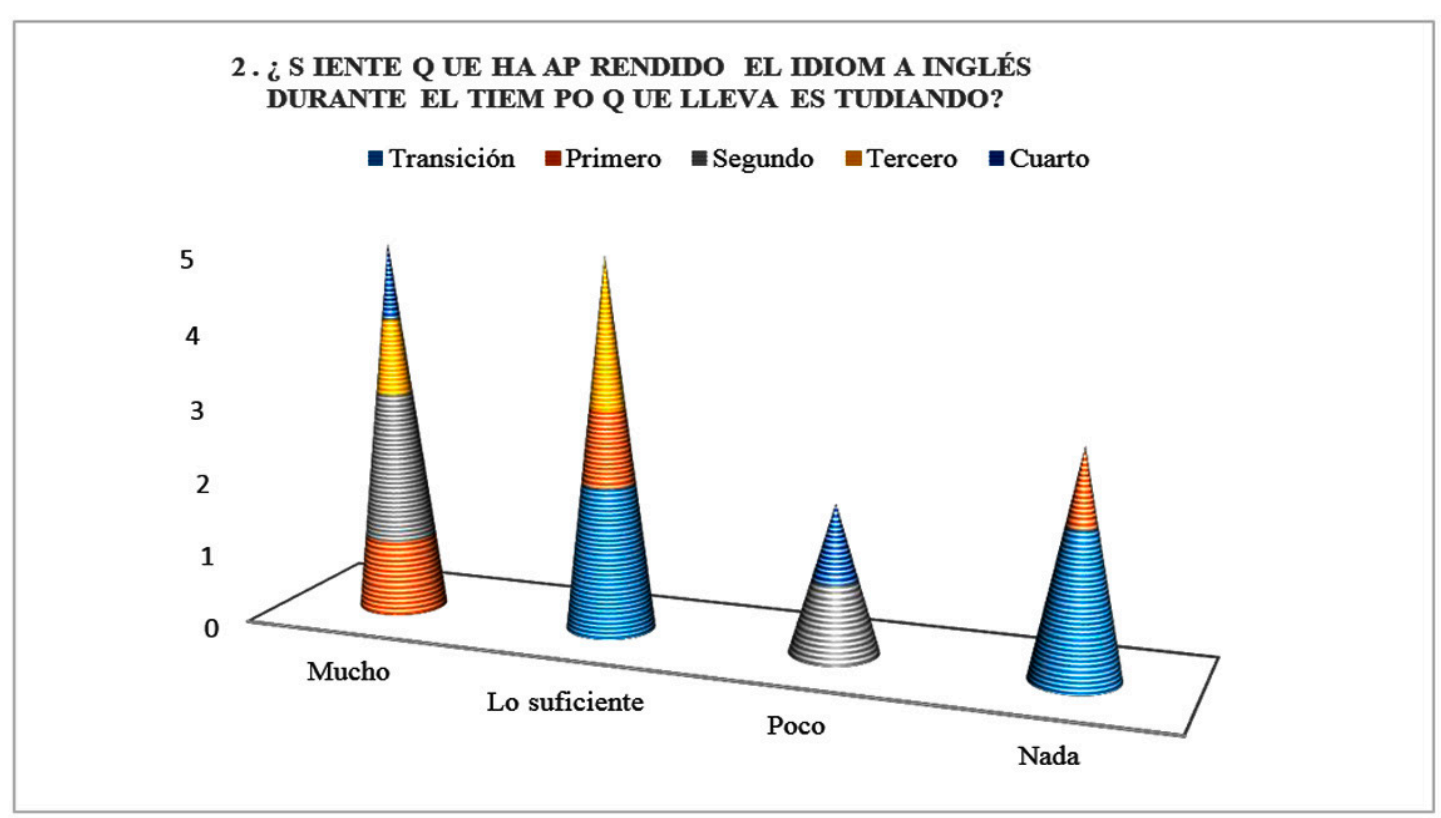

Gráfico 2. Aprendizaje. Fuente. Elaboración propia

Análisis. Con relación a la pregunta más de la mitad estuvo de acuerdo que han aprendido mucho y lo suficiente el idioma inglés durante el tiempo que llevan estudiando y una tercera parte indicaron que poco y nada han aprendido. 


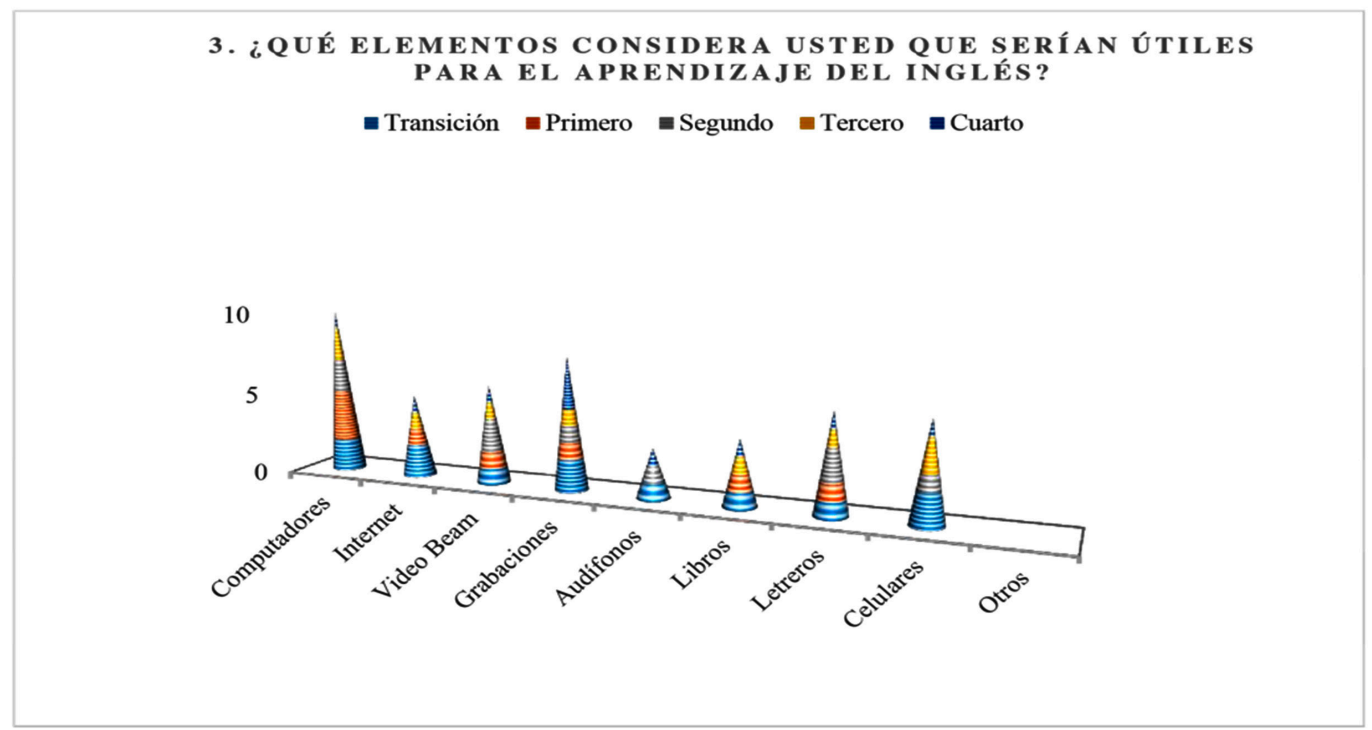

Gráfica 3. Elementos para el aprendizaje. Fuente. Elaboración propia

Análisis. Con respecto a la pregunta la mayoría estuvo de acuerdo en elegir los computadores como un elemento que sería útil para el aprendizaje del idioma inglés, seguido de las grabaciones, el video beam, celulares, los letreros, internet, libros, y una cantidad mínima los audífonos.

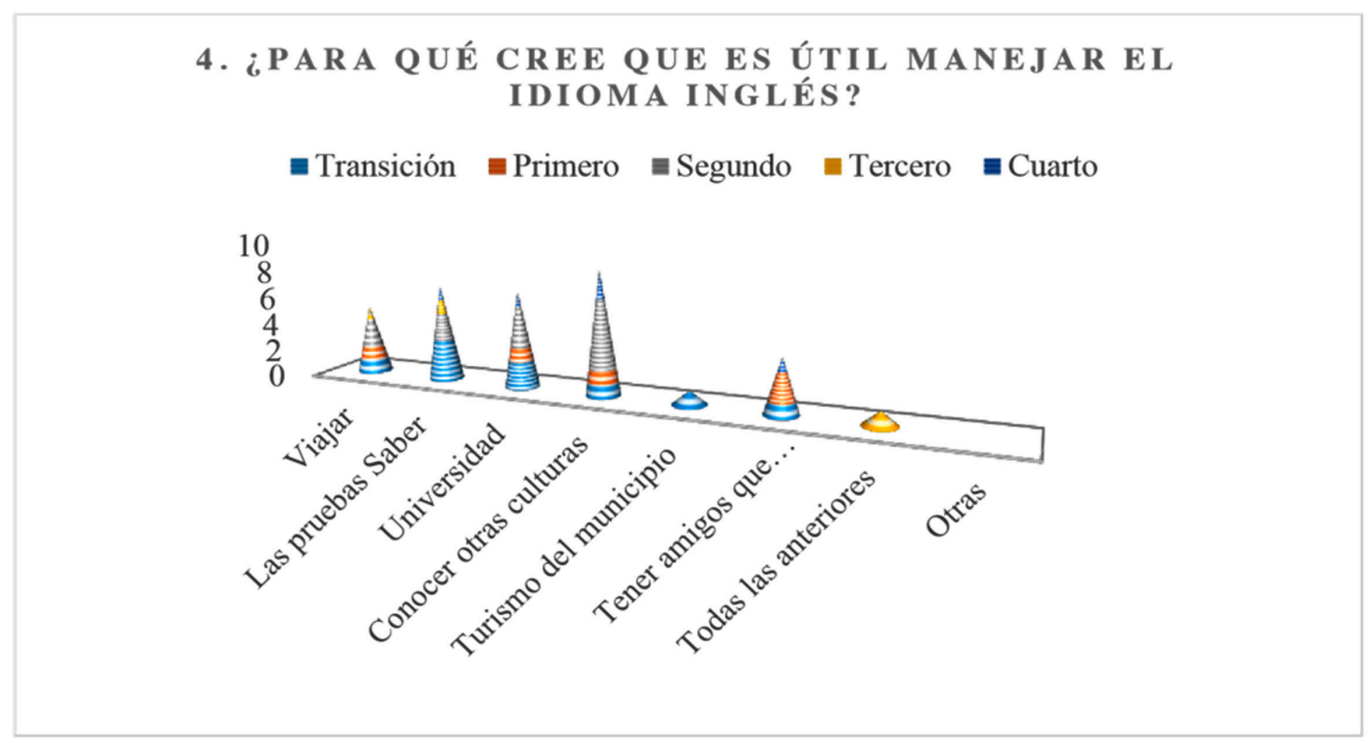

Gráfica 4. Utilidades del idioma. Fuente. Elaboración propia

Análisis. Para la pregunta más de la mitad opinaron que el idioma inglés es útil para conocer otras culturas, la mitad coincidieron en afirmar que lo es para las pruebas saber y la universidad, menos de la mitad para viajar y una cantidad mínima para el turismo. 
5. ¿CONSIDERA US TED Q UE ENCONTRAR EN S U ENTORNO ES COLAR M ENS AJES. F RAS ES, AVIS OS, ETC, CONTRIB UYE AL AP RENDIZAJ E DEL IDIOM A INGLÉS?

= Transición $\equiv$ Primero $\equiv$ Segundo $\equiv$ Tercero $\equiv$ Cuarto

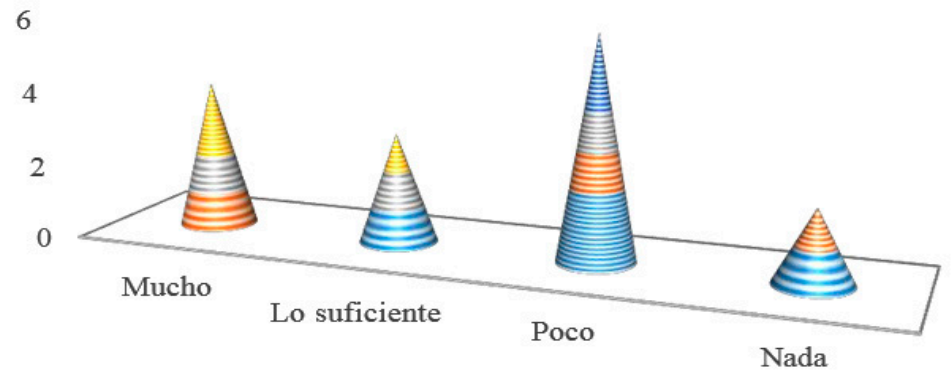

Gráfica 5. Consideraciones sobre el contexto. Fuente. Elaboración propia

Análisis. Con respecto a la pregunta, en una misma cantidad de estudiantes coincidieron en afirmar que mucho y poco contribuye encontrar en el entorno escolar mensajes, frases, avisos y en una cantidad mínima opinaron que contribuye lo suficiente.

6. ¿ CÓMO LE GUSTARÍA QUE FUERA LA CLASE DE INGLÉS ?

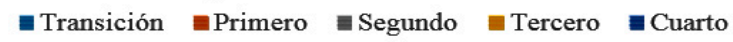

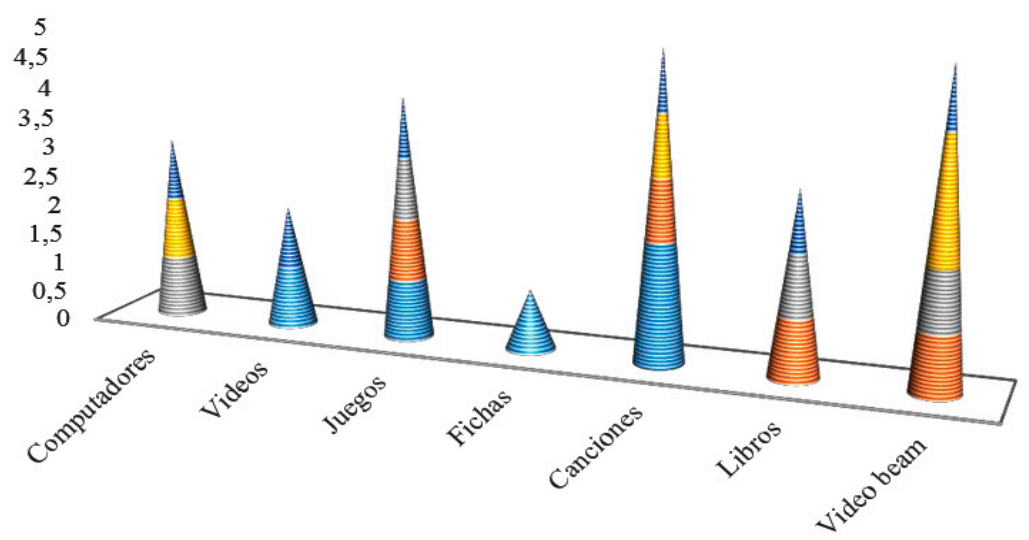

Gráfica 6. Proyecciones de la clase de inglés. Fuente. Elaboración propia

Análisis: Finalmente para esta pregunta, los estudiantes nombran algunos elementos, tienden a preferir en orden descendente: video beam, canciones, juegos, libros, computadores, videos y fichas. Lo que da como referencia que las dinámicas y las herramientas tecnológicas son de preferencia de los estudiantes. 


\section{Conclusiones Preliminares}

Los resultados de las Pruebas Saber Instruimos revelan diferencias significativas en el número de estudiantes que se encuentran en un nivel de inglés A- (no alcanzan el nivel esperado) y los que están en el nivel A1 (principiante), por tal razón buscar estrategias que mejoren el nivel de inglés de los estudiantes de esta sede, es necesario para que su desempeño aumente $y$ se obtengan mejores resultados.
Con respecto a las encuestas se puede concluirquegran partedelos estudiantes tiene gusto por el aprendizaje del idioma inglés, prefiriendo algunos medios tecnológicos como mediadores de su aprendizaje teniendo en cuenta el contexto y sus edades.

Es favorable notar que los estudiantes de esta sede son conscientes de las diferentes ventajas y utilidades del idioma inglés tanto en su vida cotidiana como en lo académico.

\section{Referencias}

Cassany, D., Valero, M., \& Vázquez, B. (2015). Desenredando la web: la lectura crítica de los aprendices de lenguas extranjeras en entornos digitales. Ocnos: Revista de Estudios sobre Lectura, (13), 07-23.

De Juan González, P. (2012). Las Nuevas Tecnologías En La Enseñanza De Lenguas Extranjera. España. Revista de Lenguas para Fines Específicos, 18, 183-212

Hernández, E. (2014). El B-Learning Como Estrategia Metodológica Para Mejorar El Proceso De Enseñanza-Aprendizaje De Los Estudiantes De Inglés De La Modalidad Semipresencial Del Departamento Especializado De Idiomas De La Universidad Técnica De Ambato. Madrid.

Jaimes, C., \& Jaimes, M. (2014). Las Tic Como Herramienta De Enseñanza Del Inglés En Las Instituciones De Educación Básica Primaria De La Región Dos.2012-2014. Pamplona

López, E., \& Londoño, L. (2012). Las Tic Como Potenciadoras En La Adquisición De Una Segunda Lengua (Tesis de pregrado). Universidad de Manizales, Colombia.

Matute, M. (2013). Uso de las tecnologías de la Información y la Comunicación en la clase de inglés de las instituciones públicas de educación secundaria del casco urbano de la Ciudad de Santa Rosa. Honduras (Tesis de maestría). Universidad Pedagógica Nacional Francisco Morazán, Honduras.

Méndez, L., Merchán, A., \& Useche, A. (2015). Las tic una alternativa para motivar 
el aprendizaje de vocabulario en ingles a los niños del grado segundo de la Institución Educativa Policarpa Salavarrieta de la vereda Tamirco del municipio de Natagaima Tolima. (Tesis de especialización). INSTITUCION UNIVERSITARIA LOS LIBERTADORES, Tolima.

MEN. (2006). Estándares Básicos de competencias en Lengua Extranjera: Inglés. Formar en Lenguas Extranjeras: ¡El reto!. Imprenta Nacional.

MEN.(2011) Programa nacional de bilingüismo: Fortalecimiento de Competencias en Lengua Extranjera, Inglés Audiovisual, ECO - English For Colombia. Bogotá.

Morchio, M. (2014). El rol de las TIC en la clase de inglés. Argentina.

Ospina Martínez, C. Las Tics Como Herramienta De Motivación En El Aula. 20082010. Bogotá

Parra, O., \& Galván, Y. (2014). Implementación de las Tic en el aprendizaje del idioma Inglés en los grados $6^{\circ}$ Institución Educativa Tomas Santos. Córdoba.

Rojas, P. (2013). Reforzando el aprendizaje del idioma inglés en el aula con el apoyo y uso de las TIC. México. 